(C) 2013. This manuscript version is made available under the CC-BY-NC-ND 4.0 license http://creativecommons.org/licenses/by-nc-nd/4.0/

\title{
Medical Diagnosis of Cardiovascular Diseases using an Interval-Valued Fuzzy Rule-Based Classification System
}

\author{
José Antonio Sanz ${ }^{* a}$, Mikel Galar ${ }^{\mathrm{a}}$, Aranzazu Jurio ${ }^{\mathrm{a}}$, Antonio Brugos ${ }^{\mathrm{b}}$, Miguel \\ Pagola $^{a}$, Humberto Bustince ${ }^{a}$ \\ ${ }^{a}$ Dept. of Automática y Computación, Universidad Publica de Navarra, Spain \\ ${ }^{b}$ Dept. of Ciencias de la Salud, Universidad Publica de Navarra, Spain
}

\begin{abstract}
Objective

To develop a classifier that tackles the problem of determining the risk of a patient of suffering from a cardiovascular disease within the next ten years. The system has to provide both a diagnosis and an interpretable model explaining the decision. In this way, doctors are able to analyse the usefulness of the information given by the system.

Methods

Linguistic fuzzy rule-based classification systems are used, since they provide a good classification rate and a highly interpretable model. More specifically, a new methodology to combine fuzzy rule-based classification systems with interval-valued fuzzy sets is proposed, which is composed of three steps: 1) the modelling of the linguistic labels of the classifier using interval-valued fuzzy sets; 2) the use of the $K_{\alpha}$ operator in the inference process and 3) the application of a genetic tuning to find the best ignorance degree that each interval-valued fuzzy set represents as well as the best value for the parameter $\alpha$ of the $K_{\alpha}$ operator in each rule.
\end{abstract}

Results

\footnotetext{
${ }^{*}$ Corresponding author. Tel:+34-948166048. Fax:+34-948168924

Email addresses: joseantonio.sanz@unavarra.es (José Antonio Sanz), mikel.galar@unavarra.es (Mikel Galar), aranzazu.jurio@unavarra.es (Aranzazu Jurio), antonio.brugos @unavarra.es (Antonio Brugos), miguel.pagola@unavarra.es (Miguel Pagola), bustince@unavarra.es (Humberto Bustince)
} 
The suitability of the new proposal to deal with this medical diagnosis classification problem is shown by comparing its performance with respect to the one provided by two classical fuzzy classifiers and a previous interval-valued fuzzy rule-based classification system. The performance of the new method is statistically better than the ones obtained with the methods considered in the comparison. The new proposal enhances both the total number of correctly diagnosed patients, around 3\% with respect the classical fuzzy classifiers and around 1\% versus the previous interval-valued fuzzy classifier, and the classifier ability to correctly differentiate patients of the different risk categories.

Conclusion

The proposed methodology is a suitable tool to face the medical diagnosis of cardiovascular diseases, since it obtains a good classification rate and it also provides an interpretable model that can be easily understood by the doctors.

Key words: Linguistic Fuzzy Rule-Based Classification Systems, Interval-Valued Fuzzy Sets, Genetic Fuzzy Systems, Cardiovascular Diseases.

\section{Introduction}

Cardio Vascular Diseases (CVDs) affect the heart and they are usually caused by some disorder that hinders the blood flow. These diseases imply a high risk of suffering from severe illness like heart attacks or thrombosis among others. They are the main health problem in adult population provoking a high death rate in many developed countries [1]. Therefore, it is important to obtain an early diagnosis of the risk of suffering from such diseases so as to start a proper medical treatment to reduce the chances of developing them.

In order to estimate such risk, Spanish doctors look up specific tables called REGICOR [2]. These tables consider different variables like gender, age, presence or absence of diabetes, systolic and diastolic blood pressure, total cholesterol and HDL cholesterol values, among others. The value provided by this procedure quantifies the risk of the patient of suffering from a CVD during the next ten years. In this manner, different categories of patients according to this value can be established. Hence, the problem of estimating the patients' risk category can be considered as a classification problem.

Fuzzy Rule-Based Classification Systems (FRBCSs) [3] are a useful tool to face classification problems. These systems are widely used because of their good performance and their capability to build an interpretable model which uses common linguistic terms for the user in the problem domain. Moreover, they offer 
the possibility of mixing information coming from different sources, i.e, expert knowledge, mathematical models or empirical measures. For this reason, FRBCSs are suitable to deal with medical diagnosis problems since, besides from providing the patients diagnosis, doctors can know the reasoning behind the decision by looking the rule or set of rules involved in the final classification.

FRBCSs use fuzzy logic [4] in order to model the linguistic terms used by the system. A key step for the subsequent success of fuzzy systems is the definition of the membership functions representing the problem information as well as possible. Sometimes, it is really difficult to determine the membership functions because the same concept can be defined in different ways by different persons [5]. This problem led Zadeh to suggest the notion of type-2 fuzzy sets [6] as an extension of fuzzy sets [4]. A particular case of type-2 fuzzy sets are the Interval-Valued Fuzzy Sets (IVFSs) [7] that assign as membership degree of the elements to the set an interval instead of a number. IVFSs allow the system uncertainties to be modelled whereas their computational effort is less than the one demanded by the use of type-2 fuzzy sets.

In [8], the authors proposed an Interval-Valued FRBCS (IV-FRBCS), that is, a FRBCS whose linguistic labels are modelled with IVFSs enhancing the performance of classical FRBCSs. In this manner, the inherent ignorance related to the definition of the membership functions was modelled by means of the IVFSs. Then, the shape of every IVFS considered in the system was optimized by using an evolutionary tuning approach. Furthermore, an Interval-Valued Fuzzy Reasoning Method (IV-FRM) was proposed, where the first two steps, namely, the computation of the matching and the association degrees for each rule of the FRBCS, used IVFSs. In order to apply the remainder of the method as in the classical FRM [9], a number was given as a result of the association degree. To compute it, the two values associated with the lower and upper bounds of the intervals were averaged, which may cause that the system does not make the most of the interval information.

In this paper, in order to handle the interval information in the IV-FRM, we introduce the $K_{\alpha}$ operator defined by Atanassov [10] to compute the association degree. In this manner, the information given by the IVFSs is exploited, since other values rather than the average one can be obtained. As a result of introducing the $K_{\alpha}$ operator, the values for the $\alpha$ parameters need to be found. In order to do so, we propose an evolutionary tuning to compute the best $\alpha$ value for each rule involved in the inference process and therefore, to provide the system with a new mechanism to take advantage of the extra information given by the IVFSs.

In the experimental study, we will show that our new IV-FRBCS allows one 
to improve the behaviour of the previous approaches when predicting the risk of suffering a CVD and hence, it allows helping the primary care doctors. The new FRBCS will only use as inputs the physical values that can be measured directly by the doctor, i.e, gender, age, smoking condition, blood pressure and body mass index. The objective of the system is to provide the doctors with a quick and reliable estimation of the patients' risk category, in such a way that they can make better decisions like deriving the patient to the secondary health centres (hospitals) or starting an appropriate treatment according to the patient's risk category if necessary.

In order to show the validity of our proposal, in the experimental study we will use two well-known FRBCSs, namely the Chi et al.'s method [11] and the Fuzzy Hybrid Genetics-Based Machine Learning (FH-GBML) algorithm [12]. We will study the behaviour of our new methodology with respect to both the initial FRBCSs and the previous IV-FRBCS [8]. To this end, we will consider the standard classification accuracy as well as the classification rate for each one of the three different CVD risk categories in which the patients can be classified.

The paper is organized as follows: the problem of the CVDs is presented in Section 2. Next, the basic concepts of IVFSs and FRBCSs along with the description of the previous proposal to combine FRBCSs with IVFSs are given in Section 3. Then, in Section 4, we describe in detail both our new proposal to introduce the $K_{\alpha}$ operator in the IV-FRM and the genetic tuning of the parameter $\alpha$. Section 5 shows the experimental framework along with the analysis of the obtained results. Finally, the main conclusions of this paper are drawn in Section 6.

\section{Problem description}

CVDs affect different parts of the body, mainly the heart and the arteries of the brain, heart and legs. Most of these diseases are induced by the decrease of either the calibre or the diameter of the arteries. The lack of blood supply does not only damage the heart but also the legs and the brain, which can lead to health disorders implying an increase of the risk of suffering from heart attacks, thrombosis or rupture of blood vessels, among others.

Among adult population, CVDs are the main health problem in general, being in the first place of the list of death cause of persons older than forty five years in many countries. As an example, about 100,000 persons per year die in Spain due to these diseases, representing a death rate of 75-150 deaths per 100,000 inhabitants depending on the region. This rate is similar in most of the developed 
countries [1]. Therefore, it is really important to estimate the patients' risk of developing a CVD in order to obtain a quick diagnosis to try to avoid their consequences.

The main CVD risk factors were identified in the Framingham Heart Study published in 1951 [13]. In Fig. 1 the different factors involved in the CVDs are depicted. Although these factors are known, their epidemiological relevance is different and, in some cases, they need extra studies so as to correctly weight them. In order to estimate the risk of suffering from a CVD, a global evaluation using the Anderson Table [14] should be made. This table is easily applicable, being advised to estimate the total coronary risk. Specifically, the following categories of risk are defined in this table:

- High CDV risk: persons having a probability greater than $25 \%$ of suffering from a CVD in ten years.

- Moderate CDV risk: the probability of a person to develop a CVD in ten years is between $10 \%$ and $20 \%$.

- Low CDV risk: the person have a probability less than $10 \%$ of suffering from a CVD in ten years.

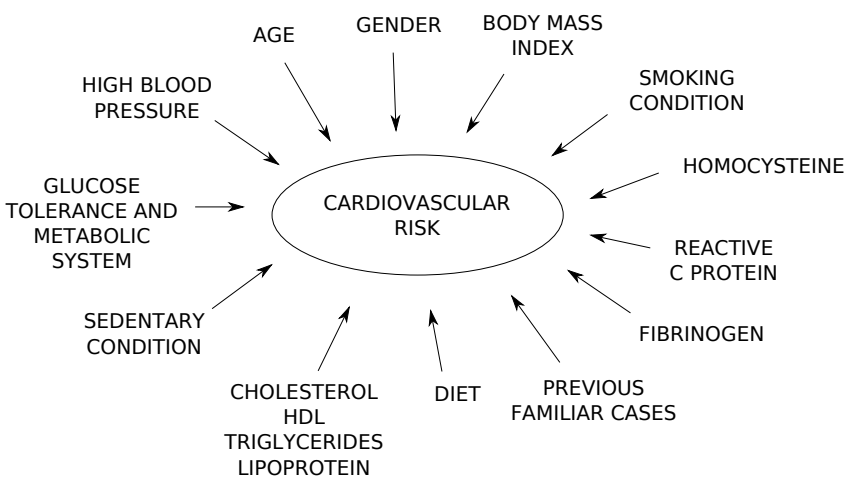

Figure 1: Cardiovascular disease involved factors.

The lower number of CVD events suffered in Spain led to develop the REGICOR tables [2], which are a version of the Anderson tables adapted to the Spanish population. Thereafter, Marrugat et al. published an estimation of the CVD risk in Spain using the calibrated Framingham equation [13]. In this paper, authors showed that in ten years, the probability of the Spanish population of suffering 
from a CVD estimated by their method is thirteen times lower than the probability computed using the Framingham approach with the patients considered as high risky when using the Framingham method. ${ }^{1}$.

In any case, the use of these tables is only advocated for primary care prevention health centres. That is, a patient derived to secondary prevention (due to previous events of ischemia, cerebral vascular or peripheral arterial accidents) has a high risk independently of the value computed using the aforementioned tables.

The patients having the main priority to be monitored and treated are those who belong to secondary prevention. They are followed by the patients belonging to primary care prevention having a bigger CDV risk, that is, patients having high, moderate and low CVD risks. This priority order makes possible:

- To avoid wasting resources of the secondary health centres due to the frequent referral of patients without an appropriate initial evaluation.

- To establish a good collaboration and coordination between the nursery services of both the primary care and the secondary care attention health centres.

- To reduce the waiting period for the first visit to the cardiovascular pathology consultation.

- To analyse the effectiveness of the therapeutic medical treatment prescribed to patients of the different groups of risk.

Furthermore, in the primary care health centres, the categorization of the patients according to their CVD risk allows to control the different risk factors affecting the patients of each category. Therefore, the main objective of the primary care doctors is to make a proper diagnosis and pharmaceutical prescription, which is adjusted to the medical evidence of each patient.

\section{Interval-Valued Fuzzy Rule-Based Classification Systems}

In this section, we first provide some preliminary concepts on both IVFSs and FRBCSs. Then, we describe the previous model that employs IVFSs to represent the linguistic labels of FRBCSs [8], which is the base for our new proposal.

\footnotetext{
${ }^{1}$ We must remark that we have to be careful with this result since the validation period of the hypothesis is still in process
} 


\subsection{Interval-Valued Fuzzy Sets}

IVFSs [15] are an extension of Fuzzy Sets [4], which was presented in 1975 by Sambuc in his doctoral thesis [7]. He applied IVFSs to deal with a medical diagnosis problem in thyroid pathology. Later, in the eighties, Gorzalczany [16] and Turksen [17] gave relevance to IVFSs, becoming definitively established. We must point out that IVFSs, which are also known as Interval Type-2 Fuzzy Sets (IT2FSs), are a particular case of Type-2 Fuzzy Sets. IVFSs have been successfully applied in classification $[8,18]$ or image processing $[19,20]$ tasks, among others.

IVFSs assigns an element of the lattice $L([0,1])$ as the membership degree of the elements to the set. $L([0,1])$ is defined as follows:

We denote by $L([0,1])$ the set of all closed subintervals of the closed interval $[0,1]$ :

$$
L([0,1])=\left\{\mathbf{x}=[\underline{x}, \bar{x}] \mid(\underline{x}, \bar{x}) \in[0,1]^{2} \text { and } \underline{x} \leq \bar{x}\right\} .
$$

We must point out that we denote $\mathbf{x}=[\underline{x}, \bar{x}]$ in order to refer to any $\mathbf{x} \in$ $L([0,1])$.

$L([0,1])$ is a partially ordered set with respect to the relation $\leq_{L}$ defined as

$\mathbf{x} \leq_{L} \mathbf{y}$ if and only if $\underline{x} \leq \underline{y}$ and $\bar{x} \leq \bar{y}$ for any $\mathbf{x}, \mathbf{y} \in L([0,1])$

$\left(L([0,1]), \leq_{L}\right)$ is a complete lattice where the smallest element is $0_{L}=[0,0]$ and the largest is $1_{L}=[1,1]$.

Once the lattice $L([0,1])$ is defined, we can recall the definition of IVFSs.

Definition 1. [16, 17, 21] An Interval-valued fuzzy set (IVFS) A on the universe $U \neq \emptyset$ is a mapping $A_{I V}: U \rightarrow L([0,1])$.

Obviously, $A_{I V}(u)=[\underline{A}(u), \bar{A}(u)] \in L([0,1])$ is the membership degree of $\mathrm{u}$ $\in U$.

There are three basic operations widely used in fuzzy set theory, namely the negation, the union and the intersection. In this paper, we use the intersection to model the conjunction among the antecedents of the rules, as usual, modelled using t-norms [22, 23, 24].

A triangular norm (t-norm) [22], $T:[0,1]^{2} \rightarrow[0,1]$, is an associative, commutative, increasing function such that $T(1, x)=x$ for all $x \in[0,1]$.

The extension of $t$-norms on IVFSs is defined as follows:

Definition 2. [21, 25] A function $\mathbf{T}:(L([0,1]))^{2} \rightarrow L([0,1])$ is said to be an interval-valued t-norm if it is commutative, associative, increasing in both arguments (with respect to the order $\leq_{L}$ ), and has the neutral element $1_{L}$. 
Definition 3. [21, 25] An interval-valued t-norm is said to be t-representable if there are two t-norms $T_{a}$ and $T_{b}$ in $[0,1]$, being $T_{a} \leq T_{b}$, so that $\mathbf{T}(\mathbf{x}, \mathbf{y})=$ $\left[T_{a}(\underline{x}, \underline{y}), T_{b}(\bar{x}, \bar{y})\right]$ for all $\mathbf{x}, \mathbf{y} \in L([0,1])$.

All interval-valued t-norm without zero divisors verify that $\mathbf{T}(\mathbf{x}, \mathbf{y})=0_{L}$ if and only if $\mathbf{x}=0_{L}$ or $\mathbf{y}=0_{L}$.

In this paper, we model the intersection of IVFSs by means of t-representable interval-valued t-norms without zero divisors that will be denoted $\mathbf{T}_{T_{a}, T_{b}}$, since they can be represented by $T_{a}$ and $T_{b}$ as defined above.

When several numerical values need to be combined into a single value we use aggregation functions $[23,24]$, which are non decreasing mappings $\mathcal{M}:[0,1]^{n} \rightarrow$ $[0,1]$ such that $\mathcal{M}(0, \ldots, 0)=0$ and $\mathcal{M}(1, \ldots, 1)=1$.

A possible way of linking IVFSs and fuzzy sets is by means of the $K_{\alpha}$ operator, which was proposed by Atanassov in 1983 [10]. This operator is applied to an interval and provide a number within the interval, depending on $\alpha \in[0,1]$. A deep study about this operator can be found in [26].

Definition 4. [10] Atanassov's operator $K_{\alpha}$ is a function $K_{\alpha}: L([0,1]) \rightarrow[0,1]$ such that

$$
K_{\alpha}(\boldsymbol{x})=\alpha \bar{x}+(1-\alpha) \underline{x} \text { with } \alpha \in[0,1] .
$$

\subsection{Fuzzy Rule-Based Classification Systems}

FRBCSs are widely used in data mining, since they allow the inclusion of all the available information in system modelling, i.e, expert knowledge, empirical measures or mathematical models. They have the advantage of generating a very interpretable model and, therefore, allowing the knowledge representation to be understandable for the users of the system. Their importance is clearly shown by their application to deal with real applications $[27,28]$. The two main components of FRBCSs are:

- Knowledge Base: it is composed of both the Rule Base (RB) and the Data Base, where the rules and the membership functions are stored respectively.

- Fuzzy Reasoning Method: it is the mechanism used to classify examples using the information stored in the knowledge base.

Any classification problem consists of $m$ training examples $x_{p}=\left(x_{p 1}, \ldots, x_{p n}, y_{p}\right)$, $p=1,2, \ldots, m$ belonging to $M$ classes where $x_{p i}$ is the $i$ th variable value $(i=$ $1,2, \ldots, n)$ and $y_{p}$ is the class label of the $p$-th training example. 
In this work, we use fuzzy rules of the following form for our FRBCSs:

Rule $R_{j}:$ If $x_{1}$ is $A_{j 1}$ and $\ldots$ and $x_{n}$ is $A_{j n}$ then Class $=C_{j}$ with $R W_{j}$

where $R_{j}$ is the label of the $j$ th rule, $x=\left(x_{1}, \ldots, x_{n}\right)$ is an $n$-dimensional example vector, $A_{j i}$ is an antecedent fuzzy set (we use triangular shaped membership functions), $C_{j}$ is a class label, and $R W_{j}$ is the rule weight [29]. In this work the rule weight is computed using the Penalized Certainty Factor (PCF) defined in [30] as:

$$
R W_{j}=P C F_{j}=\frac{\sum_{x_{p} \in \text { Class }_{j}} \mu_{A_{j}}\left(x_{p}\right)-\sum_{x_{p} \notin \text { Class } C_{j}} \mu_{A_{j}}\left(x_{p}\right)}{\sum_{p=1}^{m} \mu_{A_{j}}\left(x_{p}\right)}
$$

where $\mu_{A_{j}}\left(x_{p}\right)$ is the matching degree of the pattern $x_{p}$ with the antecedent part of the fuzzy rule $R_{j}$.

Fuzzy learning methods are the basis to build a FRBCS. In this work, we use two recognized fuzzy rule learning algorithms, i.e., Chi et al.'s rule generation method [11] and the Fuzzy Hybrid Genetics-Based Machine Learning (FHGBML) algorithm [12].

Chi et al. fuzzy rule learning method is the extension of the Wang and Mendel algorithm [31] to solve classification problems. This method is one of the most used learning algorithms in the specialized literature due to the simplicity of the fuzzy rule generation method. It generates a rule for each example in the training set following two main steps: 1) The antecedent part is composed of $n$ fuzzy sets, which are the ones associated with the linguistic label having the maximum membership degree for each variable of the problem; 2) The consequent part is composed by the label of the class of the example and the corresponding rule weight. When several rules with the same antecedent are generated, the one with the maximum rule weight is selected and the remainder ones are deleted.

FH-GBML algorithm [12] allows one to design a fuzzy rule-based system avoiding the necessity of having linguistic knowledge from domain experts, like other genetic fuzzy systems need $[32,33,34]$. The basis of the method consists of a Pittsburgh approach where each rule set is handled as an individual. It also contains a Genetic Cooperative Competitive Learning approach (an individual represents a unique rule), which is used as a kind of heuristic mutation for 
partially modifying each rule set, reaching a high search ability to efficiently find good fuzzy rules. Consequently, it generates a high quality and robust knowledge base.

Once the knowledge base of the system has been generated, a mechanism to induce the class of new examples to be classified is required. In this paper, we use the general FRM by Cordón et al [9]. Let $x_{p}=\left(x_{p 1}, \ldots, x_{p n}\right)$ be a new example to be classified, $L$ denote the number of rules in the RB and $M$ being the number of classes of the problem; then, the steps of the FRM are as follows:

1. Matching degree, that is, the strength of activation of the if-part of each rule in the $R B$ for the example $x_{p}$. A conjunction operator (t-norm) is applied in order to carry out this computation.

$$
\mu_{A_{j}}\left(x_{p}\right)=T\left(\mu_{A_{j 1}}\left(x_{p 1}\right), \ldots, \mu_{A_{j n}}\left(x_{p n}\right)\right), \quad j=1, \ldots, L .
$$

2. Association degree. The association degree of the example $x_{p}$ with the class of each rule in the $R B$.

$$
b_{j}^{k}=\mu_{A_{j}}\left(x_{p}\right) \cdot R W_{j}^{k}, \quad k=\operatorname{Class}\left(R_{j}\right), \quad j=1, \ldots, L .
$$

3. Pattern classification soundness degree for all classes. We use an aggregation function, $f$, that combines the positive degrees of association calculated in the previous step for each class.

$$
Y_{k}=f\left(b_{j}^{k}, j=1, \ldots, L \text { and } b_{j}^{k}>0\right), \quad k=1, \ldots, M .
$$

4. Classification. We apply a decision function $F$ over the soundness degrees. This function will determine the class label $l$ corresponding to the maximum value, that is, the decision function $F$ is the maximum.

$$
F\left(Y_{1}, \ldots, Y_{M}\right)=\underset{k=1, \ldots, M}{\arg \max }\left(Y_{k}\right)
$$

\subsection{Interval-Valued Fuzzy Rule-Based Classification System}

In [8], authors proposed a FRBCS whose linguistic labels were modelled by means of IVFSs to deal with the ignorance related to the definition of the membership functions. This method is composed of three steps: 1) the modelling of 
the linguistic labels by means of IVFSs; 2) the extension of the FRM to work with the new representation of the fuzzy terms and 3) the application of a genetic tuning approach to optimize the ignorance degree represented by each IVFS. In the remainder of this section we describe these steps in detail.

The first step consists of generating a knowledge base in which the linguistic labels composing the antecedent of the rules are modeled with IVFSs. To do so, an initial knowledge base is generated by means of any rule learning algorithm (e. g., the two rule learning methods introduced in Section 3.2). Then, starting from the fuzzy sets of the initial knowledge base each IVFSs is constructed as follows:

- The lower bound is the initial fuzzy set.

- The upper bound is centred at the maximum of the lower bound (being symmetrical in both sides) and the amplitude of its support is determined by the value of the parameter $W$, which initially is $50 \%$ greater than the one of the lower bound.

An example of an IVFS constructed with this procedure is depicted in Fig. 2(b). Since the IVFSs are constructed after the rule generation process, the interpretability of the initial FRBCS is maintained because the same set of rules is used.

Due to the modelling of the linguistic labels by means of IVFSs, the rule weight is compounded by a tuple $\left(\underline{R W}_{j}, \overline{R W}_{j}\right)$, whose computation is performed applying Eq. (1) considering the lower and the upper bounds respectively:

$$
\begin{aligned}
& {\underline{R W_{j}}}_{j}=\frac{\sum_{x_{p} \in{\text { Class } C_{j}}} \underline{A_{j}}\left(x_{p}\right)-\sum_{x_{p} \notin \text { Class } C_{j}} \underline{A_{j}}\left(x_{p}\right)}{\sum_{p=1}^{m} \underline{A_{j}}\left(x_{p}\right)} \\
& \overline{R W}_{j}=\frac{\sum_{x_{p} \in \text { Class } C_{j}} \overline{A_{j}}\left(x_{p}\right)-\sum_{x_{p} \notin \text { Class } C_{j}} \overline{A_{j}}\left(x_{p}\right)}{\sum_{p=1}^{m} \overline{A_{j}}\left(x_{p}\right)}
\end{aligned}
$$

where $A_{j}\left(x_{p}\right)$ and $\overline{A_{j}}\left(x_{p}\right)$ are the matching degrees of the pattern $x_{p}$ associated with the lower and the upper bounds of the IVFSs composing the antecedent part of the fuzzy rule $R_{j}$.

Since the lower bound of each IVFS is the same fuzzy set used by the rule learning algorithm, the rule weight associated with the lower bound $\left(R W_{j}\right)$ is 
equal to $R W_{j}$, which is the rule weight assigned to the rules generated by the fuzzy learning method.

The second step of the method consists of the extension of the original FRM introduced in Section 3.2 to take into account the ignorance degree represented by the IVFSs in the inference process. The two first steps are modified as follows:

- Matching degree between the antecedent of the rule and the example: We apply a t-representable interval-valued T-norm both to the lower bound and the upper bound.

$$
\begin{array}{r}
{\left[\underline{\mu_{A_{j}}}\left(x_{p}\right), \overline{\mu_{A_{j}}}\left(x_{p}\right)\right]=\mathbf{T}_{T_{a}, T_{b}}\left(\left[\underline{A_{j 1}}\left(x_{p 1}\right), \overline{A_{j 1}}\left(x_{p 1}\right)\right], \ldots,\right.} \\
\left.\left[\underline{A_{j n}}\left(x_{p n}\right), \overline{A_{j n}}\left(x_{p n}\right)\right]\right), \quad j=1, \ldots, L .
\end{array}
$$

- As association degree we take the mean between the product of the matching degree by the rule weight associated with the lower and the upper bound, respectively.

$$
\begin{array}{r}
b_{j}^{k}=\frac{\mu_{A_{j}}\left(x_{p}\right) \cdot{\underline{R W_{j}}}_{j}+\overline{\mu_{A_{j}}}\left(x_{p}\right) \cdot{\overline{R W_{j}}}_{j}^{k}}{2} \\
k=\operatorname{Class}\left(R_{j}\right), \quad j=1, \ldots, L .
\end{array}
$$

At this point a single number associated with the class is obtained and therefore, the rest of the algorithm can be applied as in the general FRM [9].

The last step of the method is the application of a tuning approach to modify the support of the upper bound of each IVFS. This is due to the fact that the same value for the parameter $W$ is used in the initial construction of every IVFS, hence, the same degree of ignorance is considered for each fuzzy term. However, the ignorance related to the definition of each membership function is probably different. For this reason, a genetic tuning proposal is applied to compute the best ignorance degree (determined by the parameter $W$ ) for each IVFS considered in the FRBCS.

The parameter $W$ takes values within the interval $[0,1]$, that is, from the situation in which both bounds are the same $(W=0)$ to the situation in which the amplitude of the upper bound is twice than that of the lower bound $(W=1)$. The amplitude of the support of the upper bound is uniformly distributed according to $W$. The noticeable situations are depicted in Figure 2. 


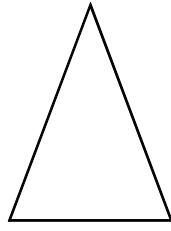

a) $W=0.0$

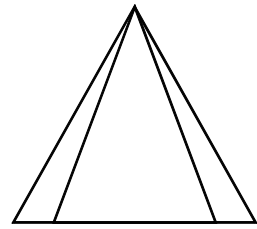

b) $W=0.5$

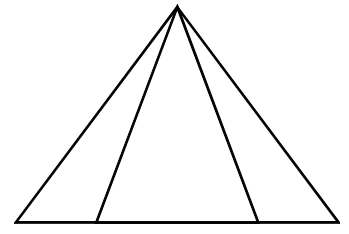

c) $W=1.0$

Figure 2: Gene values representation in the genetic amplitude tuning. a) Upper and lower bounds are the same. b) Initial construction of the IVFSs. c) The amplitude of the upper bound is twice than the one of the lower bound

\section{On the use of the $K_{\alpha}$ operator in the interval-valued fuzzy reasoning method}

This section defines our new proposal. It involves both the introduction of the $K_{\alpha}$ operator in the extended FRM with IVFSs recalled in Section 3.3 as well as the description of the genetic optimization process of the value of the parameter $\alpha$.

As we have described in Section 3.3, in [8] the first two steps of the FRM are extended in order to be able to work with IVFSs. These two steps are the computation of the matching and the association degrees (see Equations (8) and (9), respectively). In Eq. (9), the association degree is computed as the mean between the association degrees associated with the lower and the upper bounds. For this reason, we can apply the $K_{\alpha}$ operator so as to compute other values rather than the average one. Therefore, we propose to compute the association degree as follows:

$$
\begin{aligned}
b_{j}^{k}= & K_{\alpha_{j}}\left(\left[A s_{L}, A s_{U}\right]\right), \quad k=\operatorname{Class}\left(R_{j}\right), \quad j=1, \ldots, L, \\
& \text { where } A s_{L}=\min \left(\underline{A_{j}}\left(x_{p}\right) \cdot \underline{R W_{j}^{k}}, \overline{A_{j}}\left(x_{p}\right) \cdot \overline{R W_{j}^{k}}\right) \\
& \text { and } A s_{U}=\max \left(\underline{A_{j}}\left(x_{p}\right) \cdot \underline{R W_{j}^{k}}, \overline{A_{j}}\left(x_{p}\right) \cdot \overline{R W_{j}^{k}}\right) .
\end{aligned}
$$

Equations (9) and (10) are equivalent when $\alpha_{j}=0.5$, since it is the value which allows one to obtain the intermediate value of the interval. However, by setting the value of each $\alpha_{j}$ to 0.5 the information provided by the IVFSs may not be optimally exploited. Therefore, the extra information given by the IVFSs could be better handled by selecting a different value for each parameter $\alpha_{j}$, that is, adapting it for each rule. 
According to the steps of the FRM, Eq (10) is applied for each rule of the system. Therefore, the number of $\alpha_{j}$ parameters is equal to the number of fuzzy rules in the rule base, that is, $j=\{1, \ldots, L\}$. In order to try to make the best of the information provided by the IVFSs, we propose to optimize the values of the parameters $\alpha_{j}$, which can lead to improve the behaviour of the system if, indeed, there is information given by the IVFSs that was not properly handled before.

In order to accomplish this optimization problem, we consider evolutionary methods because they have proved to be appropriate tools both to deal with medical problems $[35,36]$ and to tune the values of the parameters of inference systems [37]. The tuning approach is aimed at computing both the best ignorance degree that each IVFSs represents [38] and the best value for the parameters $\alpha_{j}$ so as to provide the system with a better handling of the extra information given by the IVFSs.

We consider the use of the CHC evolutionary algorithm [39] because it provides a good trade-off between diversity and convergence, being a good choice in complex problems [40]. The components needed to design this process are explained below:

- Coding scheme: The chromosome is composed of two parts. The first part $\left(C_{A m p}\right)$ is devoted to perform the tuning of the support of the upper bound of the IVFSs, whereas the second part $\left(C_{K_{\alpha}}\right)$ is aimed at carrying out the tuning of the value of the $\alpha_{j}$ parameter for each rule. In both parts we consider the use of a real codification and their genes take values within the range $[0,1]$.

$$
\begin{gathered}
C_{A m p}=\left(W_{1}^{1}, \ldots W_{1}^{n^{k}}, \ldots, W_{n}^{1}, \ldots W_{n}^{n^{k}}\right), \\
C_{K_{\alpha}}=\left(\alpha_{1}, \ldots, \alpha_{L}\right), \\
C_{A m p+K_{\alpha}}=C_{A m p}+C_{K_{\alpha}} .
\end{gathered}
$$

where $n$ is the number of variables, $n^{k}$ is the number of linguistic labels of the $n^{t h}$ variable and $L$ is the number of fuzzy rules composing the system.

- Initial individual pool: we consider the initialization of three individuals. The first one having all the genes equal to 0.5 , which considers the original proposal of FRBCS with IVFSs, representing both the initial construction of the IVFSs and the original computation of the association degree (Eq. (9)). 
All the genes of the second and the third individuals are set to 0 and 1 , respectively, to include the extreme values. Finally, the remainder individuals are randomly initialized in $[0,1]$.

- Chromosome Evaluation: We use the most common metric for classification, i.e, the accuracy rate.

- Crossover Operator: We consider the Parent Centric BLX (PCBLX) operator, which is based on the BLX- $\alpha$ operator. Two parents are crossed if one half of their hamming distance is above a predetermined threshold, $L$. Since we consider a real coding scheme, we have to transform each gene considering a Gray Code (binary code) with a fixed number of bits per gene (BITSGENE). In this way, the threshold value is initialized as:

$$
L=(\# \text { Genes } \cdot \text { BITSGENE) } / 4.0
$$

where \#Genes stands for the total length of the chromosome. $L$ is decremented by BITSGENE when there are no new individuals in the next generation.

- Restarting approach: When the threshold value is lower than zero, all the chromosomes are regenerated at random within the interval $[0,1]$. Furthermore, the best global solution found so far is included in the population to increase the convergence of the algorithm following the elitist scheme.

\section{Experimental Study}

The experimental study aims to show the global improvement and the advantages of the application of our approach for both the patient and the health institution. To do so, we analyse the improvements achieved by the application of our new methodology with respect to the both initial IV-FRBCSs and the original FRBCS considered in this work.

We first describe the experimental framework and then, we analyse the achieved results on predicting the category of risk of the patients by studying the global accuracy rate and checking in detail the classification ability of the proposals to differentiate patients of the three categories of risk. 


\subsection{Experimental Framework}

In this section, we describe the data-set used in the generation of the FRBCS to predict the risk of suffering from a CVD along with the configuration of the fuzzy rule learning algorithms used to learn the initial rule base.

The dataset consists of 828 clinical cases obtained from the clinical records of seven primary care attention health centres of Pamplona (Navarra, Spain) during 2008. Among them, 270 cases have a high CDV risk, 281 of these patients suffer from moderate CDV risk whereas the remainder 277 cases present a low CVD risk. We have respected the Spanish Law of personal data protection (LOPD). In each clinical case, a doctor has assigned a CVD risk category following the REGICOR tables. Furthermore, the doctor also has taken into account all the data in the medical history of the patient. Therefore, some data can differ from the one recorded in the tables since doctors takes into account their own knowledge.

As input values we use the following variables:

- Gender.

- Age.

- Smoking condition.

- Blood systolic pressure.

- Blood diastolic pressure.

- Body mass index.

These variables are collected to provide a fast diagnosis tool to the doctor, since all of them can be obtained in a few minutes in a simple medical encounter. Furthermore, the provided diagnosis is objective, since the knowledge is learned from the data, and the system avoids making blood tests, which are necessary to compute the cholesterol value used to determine the risk category using the REGICOR tables.

To carry out the experiment we have considered a 10x10-fold cross-validation model (10x10-fcv), which is a standard method in classification [41]. A 10-fcv model is produced by splitting the dataset in ten equal sized folds. Then, the combination of nine of them is used to learn the classifiers and the remainder one is used to test their quality when dealing with unseen patients. This process is repeated ten times using a different test fold in each run. Therefore, after all the process all the patients will be treated as unseen cases. In the $10 x 10-f c v$ scheme, 
the 10 -fcv model is repeated 10 times using a different seed in each repetition to perform the splitting. Furthermore, for the approaches that apply a genetic tuning, we have repeated the $10 \times 10-\mathrm{fcv}$ scheme three times using a different seed for the genetic process each time. As final result, the average performance over all the testing folders is taken to measure the quality of the classifiers.

In order to compare two methods, we have used the non-parametric MannWhitney's U statistical test [42]. The logic behind this statistical procedure is to sort the results of both methods in ascending order and then assigning a rank to each result in such a way that the worst result receives the rank 1, whereas the best one receives the maximum rank (two times the number of results). If there are ties, the corresponding ranks are equally assigned. Next, for each method, the sum of the ranks is computed. As a result, if a method is regularly better than the other, the sum of its ranks will be much greater than that of the other method, which is reflected by the low p-value obtained with the test. Otherwise, if both methods provide similar results, the sum of their ranks will be similar leading to obtaining a large p-value.

Along the paper is stated that we use two different FRBCSs, namely the Chi et al. algorithm [11] and the FH-GBML method [12]. The configuration used for both FRBCSs is the same, consisting of the product t-norm as conjunction operator, the PCF to compute the rule weight and the usage of the maximum as aggregation function. Furthermore, we have considered the use of three labels per variable in the case of the Chi et al. fuzzy rule learning method, since it provides a good classification rate and a high interpretability of the final model. Regarding the FH-GBML algorithm, we have considered the following values for the specific parameters of the genetic process:

- Number of fuzzy rules: $5 \cdot d$ rules.

- Number of rule sets: 200 rule sets.

- Crossover probability: 0.9 .

- Mutation probability: $1 / d$.

- Number of replaced rules: All rules except the best-one (Pittsburgh-part, elitist approach), number of rules / 5 (GCCL-part).

- Total number of generations: 1000 generations.

- Don't care probability: 0.5 .

- Probability of the application of the GCCL iteration: 0.5.

where $d$ stands for the dimensionality of the problem (number of variables).

Finally, we have considered the following values for the parameters of the genetic tuning applied to optimize both FRBCSs: 
- Population Size: 50 individuals.

- Number of evaluations: $5000 \cdot$ number of variables.

- Bits per gene for the Gray codification (for incest prevention): 30 bits.

\subsection{Analysis of the Usefulness of the Proposed Method}

Table 1 shows the results achieved by the two learning algorithms used in this work (Chi and FH-GBML). The results obtained in training and testing are shown in the left and right part of the table respectively. In each part, the results achieved with the three different methods in this contribution are presented: the initial non interval-valued FRBCS (Base), the previous proposal to combine FRBCSs with IVFSs and genetic tuning (IVFS_Amp) and the new proposal introduced in Section 4 (IVFS_Amp $+K_{\alpha}$ ). The best result achieved in testing is stressed in boldface. We must point out that the testing results are analysed, since they show the classification ability of the classifier when dealing with unseen patients (those who have not been used in the learning process).

Table 1: Results in training and testing of both learning methods with the different approaches considered in this paper.

\begin{tabular}{|c|c|c|c|c|c|}
\hline \multicolumn{6}{|c|}{ Chi } \\
\hline \multicolumn{3}{|c|}{ Training } & \multicolumn{3}{|c|}{ Testing } \\
\hline $\begin{array}{l}\text { Base } \\
72.44\end{array}$ & $\begin{array}{c}\text { IVFS_Amp } \\
75.10\end{array}$ & $\begin{array}{c}\text { IVFS_Amp }+K_{\alpha} \\
77.29\end{array}$ & $\begin{array}{l}\text { Base } \\
70.81\end{array}$ & $\begin{array}{c}\text { IVFS_Amp } \\
72.53\end{array}$ & $\underset{\mathbf{7 3 . 8 2}}{\text { IVFS_Amp }}+K_{\alpha}$ \\
\hline \multicolumn{6}{|c|}{ FH-GBML } \\
\hline \multicolumn{3}{|c|}{ Training } & \multicolumn{3}{|c|}{ Testing } \\
\hline $\begin{array}{l}\text { Base } \\
72.72\end{array}$ & $\begin{array}{c}\text { IVFS_Amp } \\
76.47\end{array}$ & $\begin{array}{c}\text { IVFS_Amp }+K_{\alpha} \\
77.83\end{array}$ & $\begin{array}{l}\text { Base } \\
70.88\end{array}$ & $\begin{array}{c}\text { IVFS_Amp } \\
72.82\end{array}$ & $\underset{\mathbf{7 3 . 7 1}}{\text { IVFS_Amp }}+K_{\alpha}$ \\
\hline
\end{tabular}

The results of Table 1 show that our new methodology allows one to improve the classification accuracy of the initial FRBCS for both fuzzy rule learning methods. The performance of both base classifiers is increased around 3\% when applying our new method, which is a notable enhancement. Regarding the IVFS_Amp method, the improvement of IVFS_Amp $+K_{\alpha}$ is of $1.29 \%$ and of $0.89 \%$ when using the Chi et al. and the FH-GBML algorithm, respectively.

In order to stress the importance of the improvement obtained by our new approach, we have applied the Mann-Whitney's U statistical test to compare IVFS_Amp $+K_{\alpha}$ 
with the base classifier as well as with the IVFS_Amp approach. The obtained ranks and p-values for the approaches using the Chi et al. and the FH-GBML algorithms as learning methods are shown in the top and bottom part of Table 2, respectively. From these results it can be observed that regardless the algorithm used to learn the fuzzy rule base, the proposed method statistically outperforms the remainder approaches.

Table 2: Results of the Mann-Whitney's U statistical test to compare the IVFS_Amp $+K_{\alpha}$ proposal $(\mathrm{R}+)$ against the remainder classifiers (R-). The base classifier is the one defined by Chi et al.

\begin{tabular}{cccc}
\hline \multicolumn{4}{c}{ Chi } \\
\hline Comparison & Average ranks (R+) & Average ranks (R-) & p-value \\
\hline IVFS_Amp+ $K_{\alpha}$ vs. Base & 107056.5 & 73243.5 & $1.66 \mathrm{E}-15$ \\
IVFS_Amp+ $K_{\alpha}$ vs. IVFS_Amp & 97942.5 & 82357.5 & $2.42 \mathrm{E}-4$ \\
\hline \hline & FH-GBML & & \\
\hline Comparison & Average ranks (R+) & Average ranks (R-) & p-value \\
\hline IVFS_Amp+ $K_{\alpha}$ vs. Base & 105409.5 & 74890.5 & $6.54 \mathrm{E}-13$ \\
IVFS_Amp+ $K_{\alpha}$ vs. IVFS_Amp & 94985 & 85315 & 0.023 \\
\hline
\end{tabular}

Thereafter, we perform a deep analysis on the behaviour of our methodology in two specific scenarios which can provide benefits for both the patients and the medical institutions. In order to do so, we use confusion matrices [43], which allow to easily show the number of correctly classified patterns and the class in which the patterns are classified when they are misclassified. Hence, the source of the failures can be analysed.

Table 3 shows the confusion matrices of the results provided both in training (top part of the table) and testing (bottom part of the table) by the Chi et al. rule learning algorithm. The first column shows the results obtained with the classic FRBCS, the confusion matrices provided by the previous IV-FRBCS are presented in the second column and the results of our new proposal are introduced in the last column. Similarly, Table 4 presents the confusion matrices for the approaches applied using the FH-GBML rule learning algorithm. In order to obtain these confusion matrices we have summed the one hundred confusion matrices of the 10x10-fcv scheme. For the approaches that apply a genetic tuning, we have made the same process and, as final result, we have computed the average among the three confusion matrices obtained when applying the methods with the three different seeds.

The first specific scenario is the one in which the patient would have a real low risk degree of suffering a CVD. If patients are not classified with such risk degree 
Table 3: Cumulative confusion matrices in training and testing for the methods applied using the Chi et al. algorithm. The real class (R.) and the predicted class (P.) are shown in the first column and row of each matrix respectively.

\begin{tabular}{|c|c|c|c|c|c|c|c|c|c|c|c|}
\hline \multicolumn{12}{|c|}{ Training } \\
\hline \multicolumn{4}{|c|}{ Base } & \multicolumn{4}{|c|}{ IVFS_Amp } & \multicolumn{4}{|c|}{ IVFS_Amp $+K_{\alpha}$} \\
\hline R. $\mathrm{P}$. & 0 & 1 & 2 & R. & 0 & 1 & 2 & & 0 & 1 & 2 \\
\hline 0 & 23818 & 463 & 649 & 0 & 24018 & 317 & 595 & 0 & 23993 & 369 & 569 \\
\hline 1 & 5669 & 12893 & 6728 & 1 & 4461 & 14984 & 5845 & 1 & 3576 & 15524 & 6190 \\
\hline 2 & 985 & 5796 & 17519 & 2 & 893 & 6282 & 17125 & 2 & 629 & 5431 & 18240 \\
\hline \multicolumn{12}{|c|}{ Testing } \\
\hline \multicolumn{4}{|c|}{ Base } & \multicolumn{4}{|c|}{ IVFS_Amp } & \multicolumn{4}{|c|}{ IVFS_Amp $+K_{\alpha}$} \\
\hline & 0 & 1 & 2 & & 0 & 1 & 2 & & 0 & 1 & 2 \\
\hline 0 & 2619 & 63 & 88 & 0 & 2637 & 57 & 77 & 0 & 2632 & 66 & 72 \\
\hline 1 & 669 & 1348 & 793 & 1 & 584 & 1534 & 692 & 1 & 511 & 1550 & 749 \\
\hline 2 & 103 & 678 & 1919 & 2 & 103 & 750 & 1848 & 2 & 74 & 683 & 1943 \\
\hline
\end{tabular}

Table 4: Cumulative confusion matrix in training and test for the methods applied using the FHGML algorithm. The real class (R.) and the predicted class (P.) are shown in the first column and row of each matrix respectively.

\begin{tabular}{|c|c|c|c|c|c|c|c|c|c|c|c|}
\hline \multicolumn{12}{|c|}{ Training } \\
\hline \multicolumn{4}{|c|}{ Base } & \multicolumn{4}{|c|}{ IVFS_Amp } & \multicolumn{4}{|c|}{ IVFS_Amp $+K_{\alpha}$} \\
\hline $\mathrm{R} . \mathrm{P}$. & 0 & 1 & 2 & R. & 0 & 1 & 2 & & 0 & 1 & 2 \\
\hline 0 & 22337 & 1797 & 796 & 0 & 23311 & 972 & 647 & 0 & 23469 & 952 & 509 \\
\hline 1 & 3457 & 16049 & 5784 & 1 & 2945 & 16094 & 6251 & 1 & 2462 & 16878 & 5951 \\
\hline 2 & 884 & 6596 & 16820 & 2 & 575 & 5958 & 17767 & 2 & 482 & 6001 & 17817 \\
\hline \multicolumn{12}{|c|}{ Testing } \\
\hline \multicolumn{4}{|c|}{ Base } & \multicolumn{4}{|c|}{ IVFS_Amp } & \multicolumn{4}{|c|}{ IVFS_Amp $+K_{\alpha}$} \\
\hline $\mathrm{R}$. & 0 & 1 & 2 & & 0 & 1 & 2 & & 0 & 1 & 2 \\
\hline 0 & 2422 & 229 & 119 & 0 & 2500 & 167 & 103 & 0 & 2502 & 180 & 88 \\
\hline 1 & 444 & 1691 & 675 & 1 & 396 & 1641 & 773 & 1 & 350 & 1717 & 743 \\
\hline 2 & 117 & 744 & 1839 & 2 & 87 & 709 & 1904 & 2 & 72 & 727 & 1901 \\
\hline
\end{tabular}

they would not have the appropriate medical treatment, which may imply an over cost for the patients since they have to buy drugs. Regarding the primary care health centres, in this situation they suffer from a waste of resources because the monitoring of such patients is more frequent than those with a low CVD risk degree. From Tables 3 and 4 it can be observed that after the application of our new methodology the number of misclassification of patients with a low risk degree in both FRBCSs diminishes. Numerically, when using the Chi et al. algorithm as base classifier the number of misclassifications is reduced in eighteen and thirteen when applying the IVFS_Amp and IVFS_Amp $+K_{\alpha}$ methods, respectively. Re- 
garding the FH-GBML algorithm, IVFS_Amp and IVFS_Amp $+K_{\alpha}$ increases the number of correctly classified patients in seventy-eight and eighty with respect to the base classifier, respectively.

Another important situation is the one in which the patient would have a real moderate or high risk of suffering a CVD. The differentiation between both risk degrees is also important, since the monitoring frequency as well as the restrictions and medical treatments are different. From the results shown in Tables 3 and 4 , it can be noticed that the number of patients having a real moderate or high risk that are misclassified as low risk diminishes when any of the IV-FRBCSs are applied with respect to the original fuzzy system. However, we can see the following behaviour of the previous proposal using IVFS (IVFS_Amp) (when compared versus the results provided by the classical method) when classifying patients having real moderate or high CVD risks:

- Chi et al. algorithm: the number of correctly classified patients having a moderate CVD risk is increased; otherwise, the classification rate of patients having a high CVD risk is decreased.

- FH-GBML method: high CVD risk patients classification rate is improved, whereas the performance over moderate CVD risk patients is worsen.

In this scenario, it is important to observe how our new proposal (IVFS_Amp+ $K_{\alpha}$ ) allows one to improve the previously mentioned behaviour leading to enhance the performance of both classical FRBCSs for the three different classes composing the CVD diagnosis problem.

\section{Conclusion}

In this paper, we have introduced the $K_{\alpha}$ operator in the IV-FRM of a previous IV-FRBCS [8] to provide the system with a mechanism to handle the extra information given by the IVFSs. In this manner, the performance of the previous IV-FRBCS is improved, since there is interval information that was not properly exploited when applying the mean between the values associated with the lower and the upper bounds. Furthermore, we have proposed a genetic tuning method that simultaneously modify both the support of the upper bound of the IVFSs and the value of the $\alpha$ parameter for each rule.

We have applied our new methodology to tackle a medical diagnosis problem in which patients are classified according to their category of risk of suffering from a CVD. From the obtained results, it can be concluded that the use of 
IVFS $\_$Amp $+K_{\alpha}$ allows the enhancement of the classification ability of the noninterval fuzzy system for the three possible classes and, consequently, it outperforms its global performance. In addition, our new method maintains the good behaviour of the previous proposal when classifying patients having a low risk whereas it highly enhances its performance for patients having moderate or high risks. In this manner, it can be stated that our new methodology is a suitable tool to face this medical diagnosis problem because it statistically improves the remainder classifiers.

\section{Acknowledgments}

This work was partially supported by the Spanish Ministry of Science and Technology under project TIN2010-15055 and the Research Services of the Universidad Publica de Navarra.

\section{References}

[1] M. Grau, J. Marrugat, Funciones de riesgo en la prevención primaria de las enfermedades cardiovasculares, Revista Española de Cardiología 61 (4) (2008) 404-416.

[2] J. Sala, J. Marrugat, R. Masia, M. Porta, Improvement in survival after myocardial infarction between 1978-85 and 1986-88 in the regicor study, European Heart Journal 16 (6) (1995) 779-784.

[3] H. Ishibuchi, T. Nakashima, M. Nii, Classification and modeling with linguistic information granules: Advanced approaches to linguistic Data Mining, Springer-Verlag, Berlin, 2004.

[4] L. A. Zadeh, Fuzzy sets, Information and control 8 (3) (1965) 338-353.

[5] J. M. Mendel, Computing with words and its relationships with fuzzistics, Information Sciences 177 (4) (2007) 988-1006.

[6] L. A. Zadeh, Quatitative fuzzy semantics, Information Sciences 3 (1971) 159-176.

[7] R. Sambuc, Function $\phi$-flous, application a l'aide au diagnostic en pathologie thyroidienne, Ph.D. thesis, University of Marseille (1975). 
[8] J. Sanz, A. Fernández, H. Bustince, F. Herrera, Improving the performance of fuzzy rule-based classification systems with interval-valued fuzzy sets and genetic amplitude tuning, Information Sciences 180 (19) (2010) 3674-3685.

[9] O. Cordón, M. J. del Jesus, F. Herrera, A proposal on reasoning methods in fuzzy rule-based classification systems, International Journal of Approximate Reasoning 20 (1) (1999) 21-45.

[10] K. Atanassov, Intuitionistic fuzzy sets, in: VIIth ITKR Session, 1983, Deposited in the Central Science and Technology Library of the Bulgarian Academy of Sciences, Sofia, Bulgaria.

[11] Z. Chi, H. Yan, T. Pham, Fuzzy algorithms with applications to image processing and pattern recognition, World Scientific, 1996.

[12] H. Isibuchi, T. Yamamoto, T. Nakashima, Hybridization of fuzzy GBML approaches for pattern classification problems, IEEE Transactions on System, Man and Cybernetics B 35 (2) (2005) 359-365.

[13] T. R. Dawber, G. F. Meadors, F. E. Moore Jr., Epidemiological approaches to heart disease: the framingham study, American Journal of Public Health 41 (3) (1951) 279-281.

[14] K. M. Anderson, P. W. F. Wilson, P. M. Odell, W. B. Kannel, An updated coronary risk profile. a statement for health professionals, Circulation 83 (1) (1991) 356-362.

[15] H. Bustince, J. Montero, M. Pagola, E. Barrenechea, D. Gomez, A survey of Interval-Valued Fuzzy Sets, Addison Wesley, 2008.

[16] M. B. Gorlzakczany, A method of inference in approximate reasoning based on interval-valued fuzzy sets, Fuzzy Sets and Systems 21 (1987) 1-17.

[17] I. B. Turksen, Interval valued fuzzy sets based on normal forms, Fuzzy Sets and Systems 20 (2) (1986) 191-210.

[18] J. Sanz, A. Fernández, H. Bustince, F. Herrera, IIVFDT: Ignorance functions based interval-valued fuzzy decision tree with genetic tuning, International Journal of Uncertainty, Fuzziness and Knowledge-Based Systems 20 (supp02) (2012) 1-30. 
[19] H. Bustince, E. Barrenechea, M. Pagola, J. Fernandez, Interval-valued fuzzy sets constructed from matrices: Application to edge detection, Fuzzy Set and Systems 160 (13) (2009) 1819-1840.

[20] M. Galar, J. Fernandez, G. Beliakov, H. Bustince, Interval-valued fuzzy sets applied to stereo matching of color images, IEEE Transactions on Image Processing 20 (7) (2011) 1949-1961.

[21] H. Bustince, E. Barrenechea, M. Pagola, Generation of interval-valued fuzzy and atanassov's intuitionistic fuzzy connectives from fuzzy connectives and from $k_{\alpha}$ operators: laws for conjunctions and disjunctions, amplitude, International Journal of Intelligent Systems 23 (6) (2008) 680-714.

[22] K. Menger, Statistical metrics, in: Proceedings of the National Academy of Science USA, Vol. 28, 1942, pp. 535-537, (Reprinted in [Schweizer, Sklar, Sigmund, et al. (2003)] pp. 433-435).

[23] G. Beliakov, A. Pradera, T. Calvo, Aggregation Functions: A Guide for Practitioners. What is an aggregation function, Studies In Fuzziness and Soft Computing, Springer, San Mateo-California, 2007.

[24] T. Calvo, A. Kolesarova, M. Komornikova, R. Mesiar, Aggregation Operators New Trends and Applications: Aggregation operators: properties, classes and construction methods, Physica-Verlag, Heidelberg, 2002.

[25] G. Deschrijver, C. Cornelis, E. Kerre, On the representation of intuitionistic fuzzy t-norms and t-conorms, IEEE Transactions on Fuzzy Systems 12 (1) (2004) 45-61.

[26] H. Bustince, T. Calvo, B. D. Baets, J. Fodor, R. Mesiar, J. Montero, D. Paternain, A. Pradera, A class of aggregation functions encompassing twodimensional owa operators, Information Sciences 180 (10) (2010) 19771989.

[27] M.-S. Hosseini, A.-M. Eftekhari-Moghadam, Fuzzy rule-based reasoning approach for event detection and annotation of broadcast soccer video, Applied Soft Computing 13 (2) (2013) 846 - 866.

[28] G. Gowrison, K. Ramar, K. Muneeswaran, T. Revathi, Minimal complexity attack classification intrusion detection system, Applied Soft Computing 13 (2) (2013) $921-927$. 
[29] H. Ishibuchi, T. Nakashima, Effect of rule weights in fuzzy rule-based classification systems, IEEE Transactions on Fuzzy Systems 9 (4) (2001) 506515.

[30] H. Ishibuchi, T. Yamamoto, Rule weight specification in fuzzy rule-based classification systems, IEEE Transactions on Fuzzy Systems 13 (4) (2005) 428-435.

[31] L. X. Wang, J. M. Mendel, Generating fuzzy rules by learning from examples, IEEE Transactions on Systems, Man, and Cybernetics 25 (2) (1992) $353-361$.

[32] O. Cordón, F. Gomide, F. Herrera, F. Hoffmann, L. Magdalena, Ten years of genetic fuzzy systems: Current framework and new trends, Fuzzy Sets and Systems 141 (1) (2004) 5-31.

[33] F. Herrera, Genetic fuzzy systems: Taxonomy, current research trends and prospects, Evolutionary Intelligence 1 (1) (2008) 27-46.

[34] Y. Nojima, H. Ishibuchi, I. Kuwajima, Parallel distributed genetic fuzzy rule selection, Soft Computing 13 (3) (2009) 511-519.

[35] S. H. Ling, H. T. Nguyen, Natural occurrence of nocturnal hypoglycemia detection using hybrid particle swarm optimized fuzzy reasoning model, Artificial Intelligence in Medicine 55 (3) (2012) 177-184.

[36] J. Yang, H. Singh, E. L. Hines, F. Schlaghecken, D. D. Iliescu, N. G. S. Mark S. Leeson and, Channel selection and classification of electroencephalogram signals: An artificial neural network and genetic algorithm-based approach, Artificial Intelligence in Medicine 55 (2) (2012) 117-126.

[37] A. Fernández, M. J. del Jesus, F. Herrera, On the influence of an adaptive inference system in fuzzy rule based classification systems for imbalanced data-sets, Expert Systems with Applications 36 (6) (2009) 9805-9812.

[38] J. Sanz, A. Fernández, H. Bustince, F. Herrera, A genetic tuning to improve the performance of fuzzy rule-based classification systems with intervalvalued fuzzy sets: Degree of ignorance and lateral position, International Journal of Approximate Reasoning 52 (6) (2011) 751-766. 
[39] L. Eshelman, Foundations of Genetic Algorithms, Morgan Kaufman, 1991, Ch. The CHC adaptive search algorithm: How to have safe search when engaging in nontraditional genetic recombination, pp. 265-283.

[40] M. C. Lee, L. Boroczky, K. Sungur-Stasik, A. D. Cann, A. C. Borczuk, S. M. Kawut, C. A. Powell, Computer-aided diagnosis of pulmonary nodules using a two-step approach for feature selection and classifier ensemble construction, Artificial Intelligence in Medicine 50 (1) (2010) 43 - 53.

[41] J. A. Sáez, M. Galar, J. Luengo, F. Herrera, Tackling the problem of classification with noisy data using multiple classifier systems: Analysis of the performance and robustness, Information Sciences (0) (2013) -. doi:http://dx.doi.org/10.1016/j.ins.2013.06.002.

[42] H. B. Mann, D. R. Whitney, On a test of whether one of two random variables is stochastically larger than the other, The Annals of Mathematical Statistics 18 (1) (1947) 50-60.

[43] M. Galar, A. Fernández, E. Barrenechea, H. Bustince, F. Herrera, An overview of ensemble methods for binary classifiers in multi-class problems: Experimental study on one-vs-one and one-vs-all schemes, Pattern Recognition 44 (8) (2011) 1761-1776. 